\title{
Focus group study exploring the issues and the solutions to incorrect penicillin allergy-labelled patients: an antibiotic stewardship patient safety initiative
}

\author{
Neil Powell, ${ }_{1}^{1}$ Michael Wilcock, ${ }_{1}^{1}$ Neil Roberts, ${ }^{2}$ Jonathan Sandoe, ${ }^{3}$ Sarah Tonkin-Crine ${ }^{4}$
}

\begin{abstract}
'Pharmacy Department, Royal Cornwall Hospitals NHS Trust, Truro, UK

${ }^{2}$ Pharmacy Department, University Hositals Plymouth NHS Trust, Plymouth, UK ${ }^{3}$ Leeds Teaching Hospitals NHS Trust, Leeds, UK ${ }^{4}$ Nuffield Department of Primary Care Health Sciences, University of Oxford, Oxford,
\end{abstract}

\section{Correspondence to} Neil Powell, Royal Cornwall Hospitals NHS Trust, Truro TR1 3LJ, Cornwall, UK; neil. powell2@nhs.net

Received 11 January 2019 Revised 17 April 2019 Accepted 30 April 2019 Published Online First 11 June 2019

\section{EAHP Statement 5: Patient} Safety and Quality Assurance.
Check for updates

(C) European Association of Hospital Pharmacists 2021. No commercial re-use. See rights and permissions. Published by BMJ.

To cite: Powell N,

Wilcock M, Roberts N, et al. Eur J Hosp Pharm

2021:28:71-75.

\begin{abstract}
Objectives Approximately 10\% of the general population are reported to have a penicillin allergy, but more than $90 \%$ of these patients are able to tolerate penicillins after formal assessment. Patients with penicillin allergy labels have poorer health outcomes and incorrect labels impact negatively on healthcare systems. Identifying patients with incorrect penicillin allergy labels (those who can safely take penicillin) has the potential to benefit patients and healthcare systems. This study explores barriers and enablers towards identifying and removing incorrect penicillin allergy labels in inpatients ('delabelling').
\end{abstract}

Methods Two focus groups were completed with a total of 17 doctors, nurses and pharmacists at a 750-bed district general hospital in England.

Results Thematic analysis identified four main themes: managing penicillin allergic patients, environmental barriers, education for patients and staff and a future delabelling process. Staff reported that identifying and delabelling incorrect penicillin allergy records was a complex task and not a priority during the acute presentation. Participants felt confident removing erroneous allergy records if the patient was able to describe the reaction. Balancing time to confirm and delabel with competing duties was felt to be a challenge. Revisiting the discussion with the patient when time was less pressured was offered as a solution to the problem. The lack of provision to translate uncertainty about allergy status in the electronic health record was mentioned as a barrier to accurate documentation of allergy history. Ensuring all patient records were amended to reflect the new allergy status was identified as a challenge. A delabelling process involving nurses, doctors and pharmacist was discussed.

Conclusions Delabelling patients with erroneous penicillin allergy labels was recognised as a complex problem. A patient pathway involving nurses, doctors and pharmacist is likely to be the optimal method to safely delabel patients.

\section{INTRODUCTION}

Approximately $10 \%$ of the general population are reported to have a penicillin allergy, with more than $90 \%$ of these patients able to tolerate penicillins after formal allergy assessment. ${ }^{1}$ Patients with penicillin allergy labels are often prescribed second line non-penicillin antibiotics, exposing patients to an increased risk of future multidrug-resistant infections, Clostridium difficile-associated diarrhoea, treatment failure, adverse drug reactions, longer length of hospital stay and higher hospital readmission rates. ${ }^{23}$

Penicillin skin testing with oral challenge testing is the gold standard for determining whether a patient is truly allergic to penicillin. ${ }^{4}$ Due to the paucity of allergy services in the UK, skin testing is not available to many National Health Service (NHS) patients. ${ }^{5}$ However, in $20 \%-56 \%$ of patients with a penicillin allergy label, a thorough penicillin allergy history alone will identify those patients who are able to tolerate penicillin without the need for skin testing. ${ }^{6-16}$ Despite this, to our knowledge, few hospitals in England have guidelines or systems in place to delabel incorrectly labelled penicillin allergic patients (N Powell, unpublished data, 2017). There are a number of potential reasons why this may be the case. Clinicians have demonstrated a limited understanding of managing patients with a history of penicillin allergy in hospital settings. ${ }^{17}$ There are educational gaps surrounding antibiotic use in penicillin allergy-labelled patients ${ }^{18}$ as well as the lack of antibiotic allergy testing services. ${ }^{5}$

Collaboration between immunology, pharmacy and infection specialists has been postulated as a way forward ${ }^{19}$ with bedside clinical decision support and clinician education necessary to facilitate appropriate management of this patient group. 519

The aim of this study was to explore barriers and enablers towards identifying and delabelling inpatients incorrectly labelled as penicillin allergic.

\section{METHOD}

\section{Setting}

Seven hundred and fifty-bed district general hospital in England with no specialist allergy service. The hospital has a comprehensive antimicrobial stewardship programme. ${ }^{20}$

\section{Participants}

All healthcare professionals (hospital doctors, pharmacists, lead nurses and medical microbiologists on the hospital email system) were invited by email. Lead nurses were invited to nominate a staff nurse to attend the focus group. Two dates were offered in July 2017. Local medical microbiologists were unable to attend, so a medical microbiologist from a neighbouring hospital with a specialist allergy service was invited. 


\section{Focus groups}

Focus groups followed a semistructured topic guide, informed by previous work, ${ }^{20}$ which explored the scope of the problem of incorrect penicillin allergy records, the challenges in current management, potential risks to patients and possible improvements to management.

Focus groups were held in the hospital facilitated by a professional focus group moderator $(\mathrm{GH})$ and moderated and observed by the principal investigators (NP and MW). Verbal informed consent was obtained from all participants.

Proceedings were audio recorded and transcribed verbatim. The participants were known to both NP and MW through their normal duties, and all except MW and NP were unknown to GH. Participants did not receive a fee for attendance; refreshments were provided at each session.

NHS ethical approval was not required as the study did not meet the health research authority definition for research, or the requirements for NHS Research Ethics Committee approval. Patient data were used in accordance with local NHS hospital policy.

\section{Analysis}

Anonymised transcripts were analysed using thematic analysis, taking an inductive approach, by systematically coding meaningful units of data relevant to the research question. ${ }^{21}$ Coded data were grouped in terms of their similarities and differences into themes and subthemes and discussed within the research team. ${ }^{22} 23$ This resulted in a thematic framework, which captured the data across both focus groups. Data were coded initially by $\mathrm{GH}$, and the coding frame, together with subthemes, was independently assessed by NP. Regular review and discussion among authors of evolving themes contributed to data synthesis and interpretation. Minor differences in interpretation were resolved by discussion.

\section{RESULTS}

Two focus groups were completed with 17 participants: eight in the first and nine in the second. These included four consultants, four junior doctors (three foundation year 2 (FY2) (doctors in the second year of the general postgraduate medical training programme), one specialist trainee (ST1) (doctors who have completed the 2-year general postgraduate medical training programme and started their specialist training)), four nurses (a ward sister and deputy ward sister from an acute medical and acute surgical ward), four clinical ward pharmacists and one consultant medical microbiologist.

Thematic analysis identified four main themes described below.

\section{Managing penicillin-allergic patients}

For some, removing incorrect penicillin allergy labels and exposing patients to penicillin was not considered a problem. Other participants felt this was a complex issue. Participants reported inconsistencies with healthcare staff engagement with this potentially complex task. When the detail of the allergic reaction was uncertain or unknown, and especially if the patient did not require an antibiotic at that point, participants reported that it was common practice to record the reaction as 'unknown' without further investigation.

It's not so much a challenge... In clinics I ask what the allergies are if they are allergic ... what it means... I routinely say it's not an allergy, it's an intolerance. It doesn't take that long but it can be a bit of a pain. (Consultant-1)
When faced with life-threatening conditions such as sepsis, determining allergy status was also not a priority.

When is the right time to query whether a patient is allergic or not? - in the case of sepsis you only have an hour... (FY2-1)

A number of participants reported patients to be unsure about differences between being penicillin allergic or intolerant. In questioning patients' understanding of allergies, however, some felt that patients may understand the difference.

I always ask them 'have you got any allergies?' and they'll often say no and then I'll say 'have you ever had a funny reaction to medicines?' and that's when intolerances come out and then that gets recorded as allergy or intolerance. (ST1)

Use of the correct language when talking to patients was seen as important, that is, using 'intolerance' instead of 'allergy' for non-allergic side effects. Educating patients of their non-allergic status was often delivered verbally-often in the presence of the patient's family or when the patient was undergoing a procedure such as inserting a cannula.

I have de-labelled from allergy to intolerance but I would explain this in the retained notes (within the electronic prescribing system) actually patient got really bad diarrhoea. I would explain to them actually this is just your gut working really well. (Pharmacist-1)

Reassigning allergy labels was viewed as non-problematic by participants, even if the documented incorrect reaction was purported to be a severe reaction such as anaphylaxis, if the patient was able to describe the reaction. It was felt that delabelling was more difficult when patients did not want to have the label removed.

... we are ships that pass in the night so their trust is generally with their GP. They say 'the GP said this' and you are never going to change their minds. (ST1)

Gaining the confidence of patients and enabling them to consent to delabelling was also about confidence in understanding the distinction between intolerance and an allergic reaction that carries the risk of life-threatening reaction. In addition, participants felt that patients may not understand the importance of delabelling.

\section{Environmental barriers}

Balancing the time required to confirm allergy status, and delabel if appropriate, with other competing clerking duties, especially in an emergency department setting, was seen as a significant barrier.

[Y]ou don't have much time to discuss the ins and outs... balancing amount of information to record with the time available to record it... it's a time pressure thing (determining allergy status). (FY2-2)

However, for others, finding time was not an issue and asking a few questions about allergy was not perceived to be onerous.

... when you're asking a battery of clerking questions it's not that much extra work to ask what's your allergy... (FY2-3)

The notion that time should not be a barrier was expressed, and raising penicillin allergy questioning up the priority order was the challenge if a penicillin allergy confirmation and delabelling programme is to be implemented. Revisiting the discussion with the patient at a later stage of the admission and involving the ward pharmacist were deemed reasonable methods of managing time constraints.

A lack of provision to document uncertainty about an allergy status within available predetermined categories on the electronic health record was raised as a barrier. 


\section{Education for patients and staff}

Participants reported that patient empowerment was the end goal of patient education.

'I want the best antibiotic' you can say if you take penicillin you will get better quicker... the patient might say OK I'm not allergic. (Pharmacist-2)

Both groups recognised that implementing any change in the practice of delabelling patients found not to be penicillin allergic would require some form of ongoing professional development and support. The medical microbiologist raised the issues that doctors frequently enquired about prescribing for patients with penicillin allergy but when questioned about the reaction that doctor often did not know. This perception of microbiology as a ready source of information about an alternative choice to penicillin is in stark contrast to the perception by junior doctors of other sources of information they may access for other queries.

... medics don't stop to think is the microbiologist going to ask me some challenging questions, whereas with a radiologist they would expect to be asked such questions. (FY2-4)

The group acknowledged the alternative to delabelling, prescribing a second-line agent is too easy and microbiology advice too readily available. If the alternative was more difficult they felt people would try harder to establish whether the patient had a true allergy to penicillin. The imperative was for junior doctors, in particular, to establish the competence and confidence necessary to not just tick a box but to be aware of its potential consequences.

Educating junior doctors telling them the risk to that individual (who thinks they are penicillin allergic) carrying a spurious label of penicillin allergy and therefore being given gentamicin that they don't need and will give them kidney failure is far higher than any risk you are taking professionally by removing a spurious label you are making that patient safer... they should feel uncomfortable about not de labelling. (Pharmacist-3)

Another challenge raised was ensuring delabelling status is communicated widely and is amended in all patient notes in primary care, that is, general practitioner (GP), community pharmacy and care homes. However, there was some concern about whether this new status, described in a discharge letter, would be recorded on GP records and hence appear in the summary care record.

\section{A future delabelling process}

The role of nurses was considered instrumental in screening patients for possible delabelling. However, the nursing view was that any screening tool should be a prelude to a doctor when determining whether the patient is penicillin allergic.

It's not the nurse's place to question a penicillin allergy status... we need a support structure when making the decision... (Nurse-1)

Nurses reported that they should not have to make a judgement about whether a patient is allergic or not but that it was within their competences to follow a flow chart or decision aid that did not require judging a patient's status on the basis of an interpretation of the patient's responses.

I would happily do the flow chart but wouldn't feel comfortable taking the allergy off. Nurses feel comfortable asking the question but I wouldn't go around changing the EPMA (electronic prescribing medication administration system) I don't get paid enough. Nurses couldn't get into the EPMA to change anything 'We can barely prescribe two paracetamol'. (Nurse-1)
It was generally considered that any intervention based on completion of paperwork would not be an effective screening tool, partly because of the possibility of not getting the paper 'to the right place at the right time' and partly because nurses and doctors are already beleaguered by a welter of bureaucracy.

The nurses already do lots of paperwork... the trouble is nobody ever takes off any paperwork! (Nurse-2)

One suggestion for a way forward was to use the model of a dedicated nurse specialist, as with the specialist sepsis or alcohol nurse, to give support and guidance to doctors, nurses and pharmacists with the proposed programme.

Both focus groups considered the design of a possible decision aid to guide delabel decision making. Elements of an acceptable design included a standardised flow chart/questionnaire that nurses could apply to determine patient's allergy status. This would be placed in the patient's notes with the intention that a junior doctor would then enact-delabelling the patient-as appropriate. A structured pro forma would provide evidence that decision making complied with accepted practice.

Opinion was divided among the group as to whether this approach should be applied to all patients or only those requiring a penicillin.

[T] he patients you attack first are the elective ones - you have time, the patient has more time to consider and if I was short of time I would focus on those who are likely to receive antibiotics because I think I am likely to get a better interest from those patients'. (Consultant-2)

A staged process of allergy review and delabelling involving the three professions, nurses, doctors and pharmacists was agreed by the group to be a potential model. The group proposed nurses, as a more static and protocol-driven work force, to initiate the process and identify patients with a documented penicillin allergy and exclude those with a definite history of anaphylaxis. Those with incomplete history or who give a history of penicillin allergy other than anaphylaxis would be referred to the doctor. If the doctor requires more information but due to time pressures not able to find the required information, the ward pharmacist could be sought to do that.

Participants felt any intervention would need to informed by the evidence, developed by key stakeholders including microbiology and immunology and be signed off by the Trust with advanced education and healthcare worker engagement prior to implementation. Participants felt leaflets on penicillin allergy would be useful to encourage patients to ask questions about their allergy status, putting the onus on patients to start the decision tool and involving them in the decision-making process. Empowering patients to ask what the best antibiotic is for them may mean they agree to be delabelled if appropriate.

\section{DISCUSSION}

Our participants reported that managing patients with spurious penicillin allergy labels was a complex issue, and although participants reported confidence removing penicillin allergy labels if the patient gave a clear history of a non-allergic reaction, the management of these patients was inconsistent.

A need for patient education about the risks of avoiding penicillin in favour of second-line antibiotics was identified. Healthcare professionals need to be consistent with managing and describing 'penicillin allergy' if we are to increase patient understanding, a finding that has been recognised elsewhere as being crucial to advance penicillin allergy evaluations. ${ }^{24}$ Educating and empowering patients to define and manage their 
drug allergies and intolerances might lead to more penicillin allergy evaluations and delabelling. ${ }^{24}$ Determining how we might involve patients more in the process will require further studies with patients. Participants reported that they understood the risks associated with second choice antibiotics, and we have reported healthcare professional understanding of these risks elsewhere..$^{20}$ The participants recognised that healthcare professionals need postgraduate and undergraduate training enabling consistent management of incorrectly labelled penicillin-allergic patients.

Participants raised the issue of time as a barrier. Others have identified the time required to discuss allergy testing as a barrier to referring patients for allergy testing and misunderstanding among clinicians of the usefulness of the allergy history alone to determine true allergy status. ${ }^{25}$ Raising awareness of the importance of the issue of incorrect penicillin allergy records may facilitate prioritisation. If elucidating the patient's allergy history is not possible at the point of admission due to competing demand, then revisiting the issue at a less time crucial point in the patients hospital stay would still be valuable.

It is currently not possible to record a vague allergy status, for example, someone who says they have never had penicillin but that their mother had some diarrhoea. At present, the default position tends to be erring on the side of caution-recording a penicillin allergy reaction if the patient is unable to recall its precise nature.

Participants reported a lack of confidence in the communication processes between hospitals and GPs and vice versa; that is, there was uncertainty that a corrected allergy status, as part of the medicines reconciliation process, would be recorded accurately once patients are discharged back to primary care. Hence, it was felt futile to delabel a patient in secondary care because the primary care records are likely to not be updated. Putting the onus on the patient to ask about their recorded allergy and request the best antibiotic for their infection may prompt a discussion about penicillin allergy.

Increasing the barrier to prescribing second-line antibiotics might promote more appropriate prescribing. Participants felt a consistent and more robust questioning by the microbiologist when seeking antibiotic advice in penicillin allergy patients would ensure ward doctors had explored the patient's allergy history prior to seeking advice.

The participants reported that feedback on the success of any intervention to identify and delabel incorrect penicillin allergy patients would be motivating, and they also noted that there would need to be Trust-endorsed processes to protect staff making this delabelling decision.

\section{Study strengths and limitations}

This research is one of the first qualitative studies exploring healthcare professionals views and experiences of penicillin allergy delabelling. ${ }^{25}$ Participants were self-selecting, and we received a low response rate to invitations to participate likely due to workload of healthcare professionals and general availability, as such results may not be transferable to other healthcare settings. The three professions, nursing, medicine and pharmacy, were well represented though only one microbiologist attended. We were able to gain insight into the views of healthcare workers, identifying various potential barriers to implementation of an intervention to address spurious penicillin allergy labels. In addition, we were able to identify potential solutions and methods to tackle the problem that will be used to inform the next stage of the process of institutionalising a delabelling initiative.

\section{CONCLUSION}

Delabelling patients with incorrect penicillin allergy labels was recognised as a complex problem. Doctors reported erring on the side of caution and accepting allergy status with minimal questioning. The label of 'penicillin allergic' was often regarded as risk free, given the ready availability of second-line antibiotics. A greater understanding of the consequences of incorrect allergy labels is needed.

A patient pathway involving nurses, doctors and pharmacists is likely to help identify and delabel patients safely providing patient benefit.

\section{What this paper adds}

What is already known on this subject

- Delabelling patients with incorrect penicillin allergy labels has potential to improve antimicrobial stewardship practices and is expected to positively impact patients and healthcare systems.

- The benefits of delabelling incorrectly labelled patients and exposing them to penicillin instead of second-line antibiotics are widely reported, yet to our knowledge, few hospitals in England have guidelines or systems in place to do this as part of antibiotic stewardship activity.

- Here we explore the potential barriers and enablers to introducing penicillin allergy delabelling as part of inpatient care delivered by ward-based healthcare workers.

What this study adds

- Ward-based doctors, pharmacists and nurses have identified penicillin allergy delabelling as a complex task but offer a potential patient pathway to identify and safely delabel incorrect penicillin allergy-labelled patients during their inpatient stay.

Acknowledgements We would like to thank Geoff Harding for his expert guidance in the conduct of this work and for facilitating the focus groups.

Contributors All authors contributed to this work.

Funding Geoff Harding's (GH), Honorary Senior Research Fellow, University of Exeter, time was reimbursed by a Pfizer study grant and the Royal Cornwall Hospital Trust charitable funds. ST-C received funding from the National Institute for Health Research Health Protection Research Unit (NIHR HPRU) in Healthcare Associated Infections and Antimicrobial Resistance at the University of Oxford in partnership with Public Health England (HPRU-2012-10041)

Disclaimer The views expressed are those of the author(s) and not necessarily those of the NHS, the NIHR, the Department of Health and Social Care or Public Health England.

Competing interests None declared.

Patient consent for publication Not required.

Provenance and peer review Not commissioned; externally peer reviewed.

Data availability statement Data are available in a public, open access repository. There are no data in this work. Data are available on reasonable request. Data may be obtained from a third party and are not publicly available. No data are available. All data relevant to the study are included in the article or uploaded as supplementary information.

\section{REFERENCES}

1 National Institute for Health and Care Excellence. Drug allergy: diagnosis and management clinical guideline, 2014. Available: nice.org.uk/guidance/cg183

2 Moran R, Devchand M, Smibert O, et al. Antibiotic allergy labels in hospitalized and critically ill adults: a review of current impacts of inaccurate labelling. $\mathrm{Br} J \mathrm{Clin}$ Pharmacol 2019;85:492-500.

3 Blumenthal KG, Lu N, Zhang Y, et al. Risk of meticillin resistant Staphylococcus aureus and Clostridium difficile in patients with a documented penicillin allergy: population based matched cohort study. BMJ 2018;361. 
4 Mirakian R, Leech SC, Krishna MT, et al. Management of allergy to penicillins and other beta-lactams. Clin Exp Allergy 2015;45:300-27.

5 Krishna MT, Huissoon AP, Li M, et al. Enhancing antibiotic stewardship by tackling "spurious" penicillin allergy. Clin Exp Allergy 2017:47:1362-73.

6 Li M, Krishna MT, Razaq S, et al. A real-time prospective evaluation of clinical pharmaco-economic impact of diagnostic label of 'penicillin allergy' in a UK teaching hospital. J Clin Pathol 2014;67:1088-92.

7 Sigona NS, Steele JM, Miller CD. Impact of a pharmacist-driven beta-lactam allergy interview on inpatient antimicrobial therapy: a pilot project. J Am Pharm Assoc 2016;56:665-9.

8 Knezevic B, Sprigg D, Seet J, et al. The revolving door: Antibiotic allergy labelling in a tertiary care centre. Intern Med J 2016;46:1276-83.

9 Savic L, Gurr L, Kaura V, et al. Penicillin allergy de-labelling ahead of elective surgery: feasibility and barriers. Br J Anaesth. In Press 2018.

10 Trubiano JA, Smibert O, Douglas A, et al. The safety and efficacy of an oral penicillin challenge program in cancer patients: a multicenter pilot study. Open Forum Infect Dis 2018;5.

11 Mistry A, Arumugakani G, Toolan J, et al. Does de-labelling penicillin allergy lead to a respective change in primary care records? British Society Allergy and Clinical Immunology poster 2015.

12 Trubiano JA, Cairns KA, Evans JA, et al. The prevalence and impact of antimicrobial allergies and adverse drug reactions at an Australian tertiary centre. BMC Infect Dis $2015 ; 15$.

13 Preston SL, Briceland LL, Lesar TS. Accuracy of penicillin allergy reporting. Am J Hosp Pharm 1994;51:79-84.

14 Trubiano JA, Leung VK, Chu MY, et al. The impact of antimicrobial allergy labels on antimicrobial usage in cancer patients. Antimicrob Resist Infect Control 2015:4:eCollection 2015
15 Trubiano J, Phillips E. Antimicrobial stewardship's new weapon? A review of antibiotic allergy and pathways to 'de-labeling'. Curr Opin Infect Dis 2013;26:526-37.

16 Park MA, McClimon BJ, Ferguson B, et al. Collaboration between allergists and pharmacists increases $\beta$-lactam antibiotic prescriptions in patients with a history of penicillin allergy. Int Arch Allergy Immunol 2011;154:57-62.

17 Staicu ML, Soni D, Conn KM, et al. A survey of inpatient practitioner knowledge of penicillin allergy at 2 community teaching hospitals. Ann Allergy Asthma Immunol 2017;119:42-7.

18 Trubiano JA, Beekmann SE, Worth LJ, et al. Improving antimicrobial stewardship by antibiotic allergy Delabeling: evaluation of knowledge, attitude, and practices throughout the emerging infections network. Open Forum Infect Dis 2016;3.

19 Blumenthal KG, Wickner PG, Hurwitz S, et al. Tackling inpatient penicillin allergies: assessing tools for antimicrobial stewardship. J Allergy Clin Immunol 2017;140:154-61.

20 Wilcock M, Powell N, Sandoe J. A UK Hospital survey to explore healthcare professional views and attitudes to patients incorrectly labelled as penicillin allergic: an antibiotic stewardship patient safety project. Eur J Hosp Pharm 2019;26:329-33.

21 Braun V, Clarke V. Using thematic analysis in Psychology. Qual Res Psychol 2006;3:77-101.

22 Becker S, Bryman A, eds. Understanding Research for Social Policy and Practice: Themes, Methods and Approaches. Bristol: The Policy Press, 2004.

23 Pope C, Ziebland S, Mays N. Analysing qualitative data. BMJ 2000;320:114-6.

24 Blumenthal KG, Peter JG, Trubiano JA, et al. Antibiotic allergy. The Lancet 2019:393:183-98.

25 Wanat $\mathrm{M}$, Anthierens $\mathrm{S}$, Butler $\mathrm{C}$, et al. Patient and prescriber views of penicillin allergy testing and subsequent antibiotic use: a rapid review. Antibiotics 2018;7. 